la moyenne des résultats acquis sur un même modèle ( $\left.{ }^{1}\right)$.

La dernière colonne: du tableau indique la différence $d$, en pour cent, des résultats obtenus par l'emploi des types de formules.

Tous les chiffres de ce tableau sont exprimés en pieds. Le débit $Q$ se rapporte à une largeur de déversoir de un piod, et la hauteur $H$ indiquée représente la charge sur le déversoir augmentée de la charge correspondante à la vítesse d'arrivée de l'eau sur le déversoir.

Si l'on trace la courbe représentalive des variations du coefficient $\mathrm{C}$, on remarque que cette courbe s'écarte peu d'une droite pour le déversoir $n^{0} 3$, tandis qu'elle présente une forme courbo très accusée par le déversoir $n^{\circ} 4$. Pour les très faibles charges, l'influence idu bec amont du dévorsoir $n^{0} 3$ par rapport au $n^{\circ} 4$ est peu sensible, et, on tous cas, paraîtrait favoriser l'écoulement; par contre, pour les charges au-dessus de 3 pieds, olle a pour elfot de diminuer notablement la valeur du coefficient $\mathrm{C}$, et par suite celle dù débit.

\section{LA FABRICATION DU FERR0-MANGANESE AU FOUR ÉLECTRIQUE} - ( SUITE ET FIN )-

Dans le numéro précédent de La Houille Blanche, nous avons opposé le four électrique au haut fourneau pour la fabrication des iferros-manganèse. Nous voulons aujourd'hui parler des matières premières employées dans cette industrie el des usages, de jour en jour plus nombreux, de ce produit.

\section{MATIERES PREMIERES}

Minerais de manganèse. - Ils se rangent au point de vue minéralogique en deux groupes : les mincrais oxydés et hydratés d'uno part, et les minerais carbonatés et silicatés de l'aulre.

Tes minerais oxydés les plus abondants sont : la psilomélane, la polianite, la pyrolusite. La manganite, la braunite, Io haussmannite et le wad se trouvent en plus petite quantilé. - Les minerais carbonalẹ́s et silicatés, le manganèse spathique et les minerais silicieux ont une teneur plus faible en manganèse que les minerais oxydés.

On trouve les minerais de manganèse en affleurement, couches de profondeur ou métamorphisćs. Ces groupes de gittes ont beaucoup de points communs avec les couches ferrugineuses et forment avec ces dernières les groupes définis de minerais ferro-manganésifères.

Les minerais à forte teneur sont extraits principalement de la Russie; du Brésil et des Indes ; on en trouve aussi de petites quantités en Turquie, en Grèce, en France el en Espagne.

La Russie est le pays le plus riche en minerais de manganèse ; les $8 / 9$ environ de sa production provicnnent du Caucase, on en extrait aussi à Iékaterinoslaw el une pelite quantité dans l'Oural.

Dans le Ciancase, le gisement le plus important est cclui de Tschiaturi, sur le Kivirila. La région manganésifère arrosée par le Kivirila est un plateau bas partagé par de nombreuses vallées ; le sol est formé par des terrains tertiaires et crayeux, qui. reposent en profondeur sur des couches graphitiques et syénitiques. Cie minerai est dirigó sur Poti

(1) Ces coefficients ne seraient plus les memes avec l'application du syoteme métrque Rappelons que le pied vaut om 304705. et surtout sur Batoum, qui est le grand port d'expédition du minerai de manganèse pour l'Allemagne.

Au Brésil, les gisements de minerai de manganèse lés plus importants sont, d’après Kausch, ceux de la Miguel, Burnier Mine, dans le district de Minas Geraes ; de Lafayette ou Queluz, dans le voisinage de l'Ouro-Preto ; de Nazareth, dans le voisinage de San Salvador (district de Bahia) ; de Matto Grosso, au sud de Korumba ; de Macuara, dans l'Amazone, etc.

Le district brésilien le plus important au point de vue mincrai de manganèse est le Miguel Burnicr, sur le Chemin de Fer Central, près de 'l'Ouro-Preto.

Le sous-sol du pays est formé d'une couche de schistes ardoisiers d'une puissance de ro mètres; on trouve au-dessus des calcaires dolomiliques à environ $x, 50 \%$ de manganèse et, enfin, une couche de minerai de mangasèse, dont la teneur altcint $20 \%$ pour cent.

D'après Scott, il y a là deúx millions de tonnes de minerai exploitables. Ce minerai convient particulièrement bien poür la fabrication du ferro-manganèse pauvre en phosphore.

Aux Indes, la plus grande partie des minerais de manganèse provient des provinces centrales $\mathrm{d}$, en particulier, des districts de Vagpur, Balaghat, Bhandara, Chindivara et Vizagapatam, etc...

En Turquie, le gisement de Kassandra est le plus importánt. En Grèce, c'est le district minier de Milos. Le minerai de Milos est peu estimé, car il est très riche en oxyde de manganèse (Mno) silicaté, dont la réduclion est difficile. Les mincrais à faible teneur de Mn sont rares en Grèce.

La France livre des oxydes de manganèses combinés avec du carbonate et du silicate. Jc ne ferai mention que du minerai calcinć de Las Cabasses, dans le département de 1'Ariège et de celui de Romanèche.

Le domaine minier entre la Garonne et l'Ariège est composé de couches paléozoïques, étroitement pressées, orientécs. de l'ouest à l'est. Le minerai est 'du carbonate pur, en rognons ou bạtonnets, transformé en oxyde aux affleurements.

Les fosses de Las Cabasses sont situées dans la vallée de la Nert et appartiennient à la, Société "Las Cabasses Mine Ld".

Le minerai de Romanèche est un genre de psilomelane ;; sa composition est si constante que M. Lacroix, membre de l'Institut, professeur au Muséum, en a fait une espèce distincte qu'il appelle la Romanéchite, dont la formule serait.: $\mathrm{H}^{2}(\mathrm{Mn} \mathrm{Ba}) \mathrm{Mn}^{3} \mathrm{O}^{8}$.

M. Gorgen écrit cetle formule : $(\mathrm{MnBa}) \mathrm{O}, 3 \mathrm{MnO}^{2}+\mathrm{H}^{2} \mathrm{O}$. - L'eau paraît combinée.

Voici l'analyse de la Romanéchite donnée par M. Chamussy, directeur des mincs de Romanèche : $\mathrm{MnO}^{2}, 63,27 \%$; $\mathrm{Fe}^{2} \mathrm{O}^{3}, 3,50 \% ; \mathrm{SO}^{4} \mathrm{Ca}+\mathrm{Al}^{2} \mathrm{O}^{3}+\mathrm{SiO}^{2}, \mathrm{r} 3,30 \% ; \mathrm{BaO}+$ $\mathrm{CaO}+\mathrm{MgO}, \mathrm{r} 6,47 \% ; \mathrm{CO}^{2}$, corps non dosés, $3,46 \%$. Soit au total $39,94 \%$ de mangánèse métal.

La Romanéchite forme des masses compactes ou concrélionnées à texture fibreuse ; les surfaces libres des géodcs sont le plus souvent mamelonnées, stalacliformes, botrogides; elles sont quelquefois recouvertes d'aiguilles fines donnant à la surface un aspect velouté ; il cxiste des qualités pulvérulentes ; la coulcur du minẹrai est bleu d'acier ou noir ; certains échantillons présentent une cassure irisẹ́c de dịverses couleurs (queuc de paon).

Les minerais de manganèse de Romanèche étant trè̀s denses et très durs, se prêtent admirablement au traitement métallurgique ct surtout électrothermique, c'est pourquoi nous avons cru devoir leur réserver une place dáns cette étude: Son prix varie de $0,7^{5}$ ì 1,10 l'unite de manganèses. 
Nous indiquerons pour mémoire de gisement de Mincrai de Mangunèse de Huelva, en Espagne, dont l'importance va sar: cosse en diminuant.

On cxploile aussi des minerais manganésifères qui cntrent dans la composition des lits de fusion. Ils sont importés principalement d'Espagne, de Grèce et de l'Asie Mineure; en Allemagne, on utilise surtout le spath grillé de Siegen ct les minerais ferro-manganésifères de la mine de Fernic.

Voici la composition de quelques-uns de ces minerais

\begin{tabular}{|c|c|c|c|c|c|}
\hline \multicolumn{3}{|c|}{ MINERAIS A HAUTE TENEUI } & \multicolumn{3}{|c|}{ MNNERAIS FEPRO MANGANESIFERES } \\
\hline & Fe & $\mathrm{Mn}$ & & $\mathrm{Fe}$ & $\mathrm{Mn}$ \\
\hline Poti & 1,45 & 48 & Rostspat Siegerland.. & $4 \pi, 0$ & 8,5 \\
\hline Indien & 3,50 & 50,50 & Fernie bei Giessin... & 22,5 & 21,0 \\
\hline Brésil.. & 3,80 & 51,00 & fartagine (Espagne) .... & 23,0 & 19,5 \\
\hline France.. & 4,2 & 45,00 & fircer (Manganciscnstein & & \\
\hline Espagne & 2,20 & 48,30 & (saleine $) \ldots \ldots \ldots$ & 38,0 & $19,1.1$ \\
\hline
\end{tabular}

Carbone. - On emploie comme carbone le coke de gaz ordinaire et non la houille à cause de sa trop forte proportion do matières volatiles. La tencur en cendres généralement admise el qui me semble la meilleure, est de ro à $x: \%$, it condition, loulefois, que la silice soit ch fable quanlité. Ce coke sert simplement ì opérer la réduction du mincrai.

Riblons de fer. -- les riblons employés daus la mélallurgie du ferro-manganèse comprenment tous les déchets de fer, fonte, acier, tels que vis, clous, morceaux de fonte, elc. On ulilise aussi les déchets venant des Jaminoirs of plus connus sous le nom de "paille de fer des Jaminoirs".

Ces produits sonl recouverts de $\mathrm{Fe}^{2} \mathrm{O}^{3}$, en général; on ne sin préoccupe pas, mais nous croyons que la leneur en $\mathrm{fi}^{2} \mathrm{O}^{3}$ devrait être détcrminée en gros, afin de pouvoir calculcr la quantilé de coke nécessaire à la réduction de cet oxyde. La teneur moyenne cst de ro à $12 \%$.

Le prix varie de 55 à 58 franes la tonne renlue à l'usine.

Fondants. - On emploic la castine ou le fluorure de calcium (spath thor). Ces malières premières sont frop connues pour que nous nous y arrêlions.

\section{PROPRIETES ET USAGLS DES WLRROS-AANGANESE}

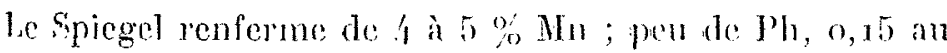
maximnom, car $\mathrm{Ph}$ rend les produils sidérurginues cassants ; peu de s, car le Mn l'ólimine; le produil présente des fimettes assez régulières ; il est blane, très dur, un peu milgiálique.

Gi-ilprès unc analyse do spiegel :. (Acier) Ledebur G $\quad$ In $\quad \mathrm{si} \quad \mathrm{P} \quad \mathrm{s}$ Spiegel petites facelles Georgs Marienhïte... 3,85 4,28 $\quad 0,37 \quad 0,09$ traces l. rayo-manganèse 46-5o \% cst hès dur, lrès compacl et lla pias cel aspect lamellaire; son grain est fin, il est blanc jamilne el présente des effets irisés. Il n'est plus du toul magnélique. A go \%, il no se lient plus el se désagrège rapidument pour tomber en poussière; plus facilcment atlapunth aux acides. II peut denfermer $-5 \%$ C quand to laux an Si cost faible.

II cit : remarquer que, plus la teneur en $\mathrm{Mn}$ augmente, plus l' point de saturation de G s'élère ; c'est ce qu'a exprimé lrès riacment $M$. Ledebur, dans un lableau que nous reproduisons ci-apiès, car il monlre bien ce phénomène d'équililire chimique.

Nin 0 io.

Point ta saturation pour le $\mathrm{C}$ corresponilant

$10-20 \quad 35 \quad 50 \quad 65 \quad 89 \quad 90$

$\begin{array}{llllll}5 & 5,5 & 6 & 6,5 & 7 & 7,3\end{array}$

En rálité, la leneur en $\mathrm{G}$ alteint même $7,5 \%$ à condition qu'il y ait très peu de Si, car le Si joue le rôle inverse du Mn.

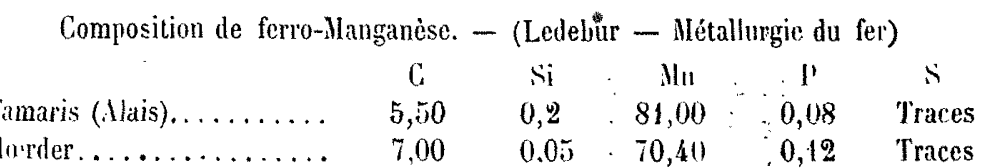

Dans la $2^{\circ}$ analyse, il y a $0,05 \mathrm{si}$ pour $7 \% \mathrm{C}$, tandis que, dans $\operatorname{la} \mathrm{I}^{\mathrm{ro}}$, contenant $0,2 \mathrm{Si}$, il y a $5,5 \% \mathrm{C}$.

Usages des ferros-manganèse. - Lics principaux usiges sont : l'affinage des aciers ordinaires ; la préparation des aciers spéciaux au manganèse ; la préparalion de certains bronzes manganeux et alliages do manganèse.

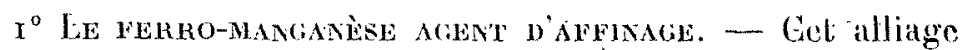
renferme en plus du fer et du mangánèse, une proportion de carbone qui varie, comme nous l'avons vu, de lf à $7,5 \%$; on conçoil done aisénent: fue ce produil servirá à mettre au point un acier. sous le rapjorl du cábone, el outre celle proprićlé, il servira ì désulfurer el à désoxyrler lo bain d'acjer. Gelle oxydation a été provoquéc, comme on le sait, par l'introduction dans le four, de la paille ide fer des laminoirs, dans Je but d'éliminer $(A-S-P$, conlenus dans les riblons et la fonte employés.

Tandis que l'oxyde de fer FeO dégage 68,9 Calories pour se former, l'oxyde $\mathrm{MnO}$ en dégage $9^{5}$, 1 ; il en résulte done que ladition du ferro-manganese a comme cónséquence la formation de $\mathrm{MnO}$ aux dépens de l'o du fer, donc agit pour désoxyder celui-ci ; en oulre, ce $\mathrm{MnO}$ contribue à scorifier le FeO non réduit el, par la sconificalion du mélange fondu, il fail disparaîle du bain lous les éléments qui s'y trouvaient. unis ou dissous : c'est là sa principale aclion d'aflinage. De plus, il résulte de la désoxydation, un bain prive de gaz, ce qui évile, par suile, les soufflures.

C'est en se basanl sur ce demier phénomène, que ecr. taines aciéries Martin, pour éviter les soufllures, introduisaient du ferro-manganèse dans la poche de coulée ellemême. Ce procédé est sujet à controverse, car s'il fait disparaitre less soufflures, il foumit in acice " non homogène".

le $\mathrm{Fe}^{2} \mathrm{O}^{3}$, e FoO existant dans le bain, ne peuvent plus s'éliminer, puisque loule la Sio² est dójà éliminéc, mais le ferro-manganese par soin Mn va s'oxyder aux dépens du

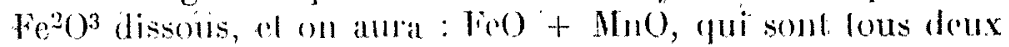
bing plus atsenent seoriliables.

Ensuite, le fere agira par son ci el, si la juoportion en a élé bien calculé, on arrivera très lacilement à lacier voulu.

On a essayé de recarburer directement avec $c$, mais on a une perle énorme en $($, paree quime partic avanl de se dissoudre dons le bain, brùle à la surface, landis yue le ferro, qui ronforme deja le (i dissous, s'assimile plus facilement.

Introduction du ferro-Mn ilans les aciers. - L'introduclion du ferro-manganèse dans les aciers se fait suivant différents procédés. 1)'ordinaire, elle so fail pen dë lemps (environ une demi-heure) avant, la eoulée.

Dans le four Brssomer, si l'on ajoule du spiegel (forro-Mn an-dessous de $95 \% \mathrm{Mn}$ ), comme il en faul une ruantité assez forte, l'introduclion se fait sous forme de produit fondu, afin de ne pas refioidir le bain.

Si on emploie du ferro-manganese proprement dit, le produit s'ajoute sous forme solide au moment précis où la scorification, c'està-dire la disparition, de si du bain, est terminéc. Le monent d'introduclion du forro es conmu sous lo nom de période des fumées. Aussitôl le ferro jntroduit, il se produit un bouillonnement intense du bain qui cesse quand les réaclions sont terminées. 
Dans le four Martin, l'addition du spiegel ou du ferromanganèse se fait presque à la fin de l'opération, quand $\mathrm{SiO}^{2}, \mathrm{~S}$ et $\mathbf{P}$ sont éliminés ; on détermine par un essai physique sur une éprouvette, la teneur en $\mathrm{C}$ et on ajoute alors Je ferro; on le projette en morceaux de la grosseur d'une noix à la surface dı bain ; le soufllage des gaz opère le mélange avec le bain.

Dans les fours électriques, l'introduclion de ferro-manganèse se fait toujours 30 à 25 minutes avant la coulée. On soulève une des portes du four et on projette le produit à la pelle à la surface du bain; gralce à la température élevéc te ces fours, le bouillontrement de l'acier produit lo mélange intime du bain et du ferro.

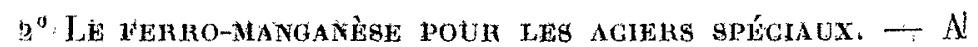
partir de $0,25 \mathrm{Mn} \%$ o, on a les aciers au $\mathrm{Mn}$, très peu étudiés encose, tnafis dont l'emploi se généralise de plus en plus. Is tendent it remplacer les aciers au nickel dans la plupart de leurs utilisations el, cela, poir deux raisons : leur prix moins thevé, leurs propriétés spéciales.

Cés aciers offrent une grande résistance au choc. Lor'sque lit proportion de Mn varie de $x$ à $5 \%$, l'augmentation de fu chatge de rupture et Ia limile dastique oroissent lentement, et la striclion et les allongéments diminuent ; c'est ve qui a fait próeoniser l'acier au Mn pour la fabrication des rails et des aiguillages des chemins de fer.

Un adier fait au Creusol, et qui renfermait : C, x à $\mathrm{x}, 5 \%$, Mil, xa à $15 \%$, trempé à l'eau à $900^{\circ}$, a présenté les caracléristiques stuivantes qui donnesit raison aux considérations d-dessus exposées :

$$
\mathrm{K}=95 \mathrm{kggs} \mathrm{pat}^{\mathrm{i}} \mathrm{minh}^{3} ; \quad \mathrm{E}=35 ; \quad \mathrm{A}=45 \quad\left({ }^{1}\right) ;
$$

ators qu'un acier extra dur, ayant o, $8 \%$ de $\mathrm{C}$, a pour caractéristiques : $\mathrm{R}=85$-100 kgs $\quad \mathrm{E}=55 ; \mathrm{A}=8$.

("est ce qui justifie le mouvement actucl qui tend à donnor li prótérenee aux aciers manganésés contre les aciers au nickel. Une retison imporlante et qui, nous l'espérons, interviendra pour une large part dans cette lutte, sera celle du bas prix do revient l'un acier an Mn par rappotl à un acion au Ni. Il nous a paru intéressant, en outre, de ciler ci-après, quelques chiffres extraits d'un article de MM. Schneider et $\mathrm{C}^{10}$, paru dans la Revue de Métallurgie de mai 1 gog.

Là résistance de l'acier au Mn est plus élevéc ruc ed le a $20-2 \% \% \mathrm{Ni}$; clle dépasse 100 kilogs. Propriétés, en somure, comparables à celles des aciers au tuingstène, niais, conme arantage sul ces derniers, l'acier au Mn n'est pas si fragile.

\section{ESSAIS DE TRACTION}

Barreaux de classement de $16 \mathrm{~mm}$. de diamètre ; longueur entre repères : $100 \mathrm{~mm}$. ; forgés et trenıpés.)

\begin{tabular}{|c|c|c|c|c|c|}
\hline Hereres entre barreatux & $\begin{array}{c}\text { limite } \\
\text { d'é̉asticitité } \\
\text { F }\end{array}$ & $\begin{array}{l}\text { Clararge de } \\
\text { ruptiore } \\
\text { a par urme }\end{array}$ & $\begin{array}{c}\text { Allongerment } \\
.1 \%\end{array}$ & $\begin{array}{l}\text { Contraction. } \\
1-\frac{S}{s}\end{array}$ & OBSEMVITTONS \\
\hline 1 & $\$ 1.00$ & 19.60 & 44,00 & 0,440 & Cassure is lieri \\
\hline 2 & 43,70 & 89,60 & 46,00 & 0,437 & an silfet \\
\hline 3 & 40,60 & 97,20 & 42.00 & 0,395 & \\
\hline 4 & 41,80 & 107,80 & 55,00 & 0.425 & \\
\hline 5 & 39,20 & 103,40 & 48,50 & 0,421 & \\
\hline 6 & 39,20 & 93,80 & $4,5,00$ & 0,415 & \\
\hline 7 & 42,80 & 105,40 & 51,00 & $0,4,3$ & \\
\hline 8 & 41,40 & 102,20 & 54,00 & 0,430 & \\
\hline
\end{tabular}

$\Lambda$ titre de comparaison, nous donnons des chiffres sur les teier au $\mathrm{Ni}$, qui peuvent servir de point de comparaison

(i) $R=$ rupturè à lia traction pár millimètre carré ; $\mathrm{E}=$ limite ćlastique; $A=$ allongement pour cent. zvec les acier's au Mn. - Pour une leneur en Ni égale à une teneur en Mri, on a des acicrs moins résistants.

Acietis AU Ni (Ledebur).

\begin{tabular}{|c|c|c|c|c|c|c|c|c|c|}
\hline $\mathrm{Ni}$ & G & $\mathrm{Si}$ & $s$ & $\mathrm{p}$ & In & $\begin{array}{l}\text { Rupture } \\
\text { par mal" }\end{array}$ & $\begin{array}{l}\text { Limite } \\
\text { dè̉'esticité }\end{array}$ & $\begin{array}{l}\text { Allunge- } \\
\text { ment } \%\end{array}$ & $\begin{array}{c}\text { Striction } \\
\%\end{array}$ \\
\hline 0,27 & 0,19 & 0,31 & 0,10 & 0,09 & 0,79 & 49,5 & 26,8 & 35,6 & 55,9 \\
\hline 1,92 & 0,14 & 0,21 & 0,08 & 0,07 & 0,71 & 54,2 & 54,2 & 40,8 & 55,2 \\
\hline 5,81 & 0,18 & 0,31 & n. $\mathrm{d}$. & - & 0,65 & 64,4 & 44,7 & 30.8 & 55,2 \\
\hline 9,51 & 0,18 & 0.20 & 一 & - & 0,86 & 134,2 & 70,0 & 0,3 & 54,2 \\
\hline 15,48 & 0.23 & 0,34 & 0,11 & - & 0,63 & 147,6 & 81,6 & 3,2 & 2,5 \\
\hline 10,64 & 0,10 & $0,2 i$ & B. d. & n. $d$. & 0,93 & $142 ; 8$ & 74,8 & 6,9 & $.6,4$ \\
\hline 29,07 & 0,14 & 0,38 & - & - & 0,85 & 60,4 & 39,2 & 32,9 & 45,0 \\
\hline
\end{tabular}

\section{CONCLUSION}

Elant donné tous les avantages de ces ferro-alliages qui constituent des produits de haute valeur marchande, nouls sonmes convainicus que le mouvenent qui tend à donner la préférence au ferro-manganèse électrique, s'affirmera de pilùs en plus et que, grâce à nos belles chutes des Alpes, nous arriverons ì la fabrication de la presque tolalité des alliages de fer el de manganèse dans les usines électrónélallurgiques.

On aura ainsi réalisé un progrès. En effet, pauvres en charbon, mais riches en houille blanche, nous monopolis serons chez nous celte métallurgic des métaux spéciaux qui compensera, par le haul prix des produits, le chiffre d'affaires que nos rivaux industriels tendent a nous chlever palr Jes gros tonmages dans ta fabricalion des produits comniuns. Quand on voit comment la houille blanche a bien justifié les espérances qu'elle a fait naître, on est confiant danis l'avenir. Nous souhailons que, pour la France, cette branche do l'électrométallurgic continue à justifier le titre "d'industric nationale " qu'on lui a si justement donné.

Charles GAY,

Ingénicu-chimiste (électrométallurgiste), Licencié ès-scicuces.

\section{GONSTRUGTION DE LACS ARTIFIGIELS EN SARDAIGNE ET DANS TA GALIBRE}

Ia Chambre des Députés italienne vient d'upprouver un projet de loi pour la crćation de grands laes artificiels sur les fleuves : Sila, en Cadabre, et Tirso, en Sardaigne.

II s'agit d'installations et constructions colossales n'ayant pas de précédents en Europe. Sur Ja Sila, on construira trois réservoirs qui fourniront au total 150000 chevaux, pouvant ctre employés pour traction, éclairage et force motrice en Calabre, dans les Pouilles et dans toul le midi. Le devis est de 65 millions. Les installations de la Sila serviront également à arroser une vaste plaine du Cotronese.

Le réservoir du Tirso, en Sardaigne, coûtera 25 millions de lires et pourra contenir 330 millions de mòtres cubès avec un périmètre de 6o kilomètres environ. Il servira șirtout pour litrigation de 20 ooo hectares de terrain, et produira quelques nilliers de chevaux qui pourront être utilisćs dans les mincs de l'île.

Pour ces deux grandioses installations, l'industrie privéc ne demanderait à l'Etal que les facililés fiscales accordées par les lois édictées pour le Midi.

Les concessionnaires devront tenir à la disposition de l'Etat et au prix de revicnl, l'énergie nécessaire pour los Services publics et, après 60 années, au terme de la concéssion, les réservoirs, les centrales et les installations principalles passeront gratuitement à l'Elat. 